\title{
Method of Lower and Upper Solutions for Elliptic Systems with Nonlinear Boundary Condition and Its Applications
}

\author{
Ruyun Ma, Ruipeng Chen, and Yanqiong Lu \\ Department of Mathematics, Northwest Normal University, Lanzhou 730070, China \\ Correspondence should be addressed to Ruyun Ma; ruyun_ma@126.com
}

Received 8 March 2014; Accepted 30 April 2014; Published 12 May 2014

Academic Editor: Wan-Tong Li

Copyright ( 2014 Ruyun Ma et al. This is an open access article distributed under the Creative Commons Attribution License, which permits unrestricted use, distribution, and reproduction in any medium, provided the original work is properly cited.

We develop the method of lower and upper solutions for a class of elliptic systems with nonlinear boundary conditions. As its application, an elliptic system modeling a population divided into juvenile and adult age groups is studied, and we find sufficient conditions in terms of the principal eigenvalue of the corresponding linearized system, to guarantee the existence of coexistence states of the above juvenile-adult model.

\section{Introduction}

Let $\Omega$ be a bounded domain in $\mathbb{R}^{N}(N \geq 2)$ with sufficiently smooth boundary $\Gamma$. In this paper we study the elliptic systems of the form

$$
\begin{array}{r}
L_{k} u_{k}=f_{k}\left(x, u_{k},[u]_{k}\right), \quad x \in \Omega, \\
\frac{\partial u_{k}}{\partial \mathbf{n}}+b_{k}(x) u_{k}=g_{k}\left(x, u_{k},[u]_{k}\right), \quad x \in \Gamma, \\
k=1,2, \ldots, m,
\end{array}
$$

where $\mathbf{n}$ is the unit exterior normal to $\Gamma, u=\left(u_{1}, \ldots, u_{m}\right)$, $[u]_{k}=\left(u_{1}, \ldots, u_{k-1}, u_{k+1}, \ldots, u_{m}\right)$, and $L_{k}(k=1,2, \ldots, m)$ is a linear differential operator of the form

$$
\begin{aligned}
L_{k} u= & -\sum_{i, j=1}^{N} a_{i j}^{k}(x) D_{i} D_{j} u \\
& +\sum_{i=1}^{N} a_{i}^{k}(x) D_{i} u+a^{k}(x) u .
\end{aligned}
$$

with symmetric coefficient matrix $\left(a_{i j}^{k}\right)_{n \times n}$. We suppose that $a_{i j}^{k} \in C^{2+\alpha}(\bar{\Omega}), a_{i}^{k} \in C^{1+\alpha}(\bar{\Omega})$, and $a^{k} \in C^{\alpha}(\bar{\Omega})$ for a certain $\alpha \in(0,1)$. Moreover, $L_{k}(k=1,2, \ldots, m)$ is supposed to be strongly uniformly elliptic; that is, $\sum_{i, j=1}^{N} a_{i j}^{k}(x) \xi_{i} \xi_{j} \geq$ $\alpha_{0}^{k}|\xi|^{2}$ for some constant $\alpha_{0}^{k}>0$ and every $x \in \Omega$, $\xi=\left(\xi_{1}, \ldots, \xi_{N}\right) \in \mathbb{R}^{N}$.

System (1) with $m=1$ arises, in particular, in the study of steady state solutions of nonlinear parabolic equations of the form

$$
\begin{gathered}
\frac{\partial u}{\partial t}+L u=f(x, u) \quad \text { in } \Omega \times(0, \infty), \\
\frac{\partial u}{\partial \mathbf{n}}+b(x) u=g(x, u) \quad \text { on } \Gamma \times(0, \infty), \\
u=u_{0} \quad \text { on } \bar{\Omega},
\end{gathered}
$$

where $L$ is some second order, strongly uniformly elliptic differential operator. In this connection, nonlinear boundary conditions seem to be of particular importance. For the study of the stability of the solutions of the parabolic initial-boundary value problem (3), one has to have a good knowledge of the steady states, that is, of the solutions of (1) with $m=1$. In the past few decades, the theory of monotone operators has been applied to boundary value problems of the form (3); see, for example, [1-3] and the references therein. In all of the above-mentioned papers, the boundary condition is of the special form $\partial u / \partial \mathbf{v}=g(u)$, where $g$ is decreasing and $\mathbf{v}$ is the conormal with respect to the differential operator $L$. 
Besides these results, there are some scattered existence theorems for nonlinear Stecklov problems of the form

$$
\begin{aligned}
& L u=0 \quad \text { in } \Omega, \\
& \frac{\partial u}{\partial \mathbf{v}}=g(x, u) \text { on } \Gamma,
\end{aligned}
$$

where $L$ is supposed to be formally self-adjoint such that the homogeneous linear boundary value problem possesses a nontrivial solution; we refer the readers here to $[4,5]$ and the references therein. The stationary version of (3), which covers the above-mentioned several situations, has been studied by several authors; see Amann $[6,7]$ and Hess [8]. In particular, Amann [6] studied the stationary version of (3) and obtained a general existence theorem for it, namely, the result that the existence of a subsolution and a supersolution guarantees the existence of a solution. By transforming the stationary version of (3) into an equivalent fixed point equation in $C(\bar{\Omega})$, he gave a new and more elegant proof for the above result.

Motivated by the above work, we will develop the method of lower and upper solutions for system (1) under the following assumptions:

(H1) for $k=1, \ldots, m, f_{k}: \bar{\Omega} \times \mathbb{R}^{m} \rightarrow \mathbb{R}$ is $\alpha$-Hölder continuous in the first variable and locally Lipschitz in the second variable; $g_{k}: \Gamma \times \mathbb{R}^{m} \rightarrow \mathbb{R}$ is locally Lipschitz continuous;

(H2) For $k=1, \ldots, m, a^{k}>0$ in $\Omega$, and $b^{k} \in C^{1+\alpha}(\Gamma)$ satisfying $b^{k}>0$ on $\Gamma$.

By a solution $u=\left(u_{1}, \ldots, u_{m}\right)$ of (1) we mean a classical solution, that is, for each $k=1,2, \ldots, m, u_{k} \in C^{2}(\Omega) \cap C^{1}(\bar{\Omega})$ such that $L_{k} u_{k}(x)=f_{k}\left(x, u_{k}(x),[u]_{k}(x)\right)$ for $x \in \Omega$, and $\partial u_{k}(x) / \partial \mathbf{n}+b_{k}(x) u_{k}(x)=g_{k}\left(x, u_{k}(x),[u]_{k}(x)\right)$ for $x \in \Gamma$. For given $v=\left(v_{1}, \ldots, v_{m}\right)$ and $w=\left(w_{1}, \ldots, w_{m}\right)$, we say $v \leq w$ if $v_{k} \leq w_{k}$ for every $k=1,2, \ldots, m$, and we define

$$
\begin{gathered}
{[v, w]=\left\{u \in[C(\bar{\Omega})]^{m}: v_{k}(x) \leq u_{k}(x) \leq w_{k}(x),\right.} \\
\forall x \in \bar{\Omega}, k=1,2, \ldots, m\} .
\end{gathered}
$$

Definition 1. Let $\bar{u}, \underline{u} \in\left[C^{2}(\Omega) \cap C^{1}(\bar{\Omega})\right]^{m}$. Then $\bar{u}$ and $\underline{u}$ are called ordered coupling upper and lower solutions of $(1)$, if $\bar{u} \geq \underline{u}$ and they satisfy

$$
\begin{gathered}
L_{k} \bar{u}_{k} \geq f_{k}\left(x, \bar{u}_{k},[u]_{k}\right), \\
\forall[\underline{u}]_{k} \leq[u]_{k} \leq[\bar{u}]_{k}, x \in \Omega, \\
\frac{\partial \bar{u}_{k}}{\partial \mathbf{n}}+b_{k}(x) \bar{u}_{k} \geq g_{k}\left(x, \bar{u}_{k},[u]_{k}\right), \\
\forall[\underline{u}]_{k} \leq[u]_{k} \leq[\bar{u}]_{k}, x \in \Gamma, \\
k=1,2, \ldots, m, \\
L_{k} \underline{u}_{k} \leq f_{k}\left(x, \underline{u}_{k},[u]_{k}\right), \\
\forall[\underline{u}]_{k} \leq[u]_{k} \leq[\bar{u}]_{k}, x \in \Omega,
\end{gathered}
$$

$$
\begin{array}{r}
\frac{\partial \underline{u}_{k}}{\partial \mathbf{n}}+b_{k}(x) \underline{u}_{k} \leq g_{k}\left(x, \underline{u}_{k},[u]_{k}\right), \\
\forall[\underline{u}]_{k} \leq[u]_{k} \leq[\bar{u}]_{k}, x \in \Gamma, \\
k=1,2, \ldots, m,
\end{array}
$$

respectively.

The main result of this paper is the following.

Theorem 2. Assume that (H1) and (H2) are satisfied. Let $\bar{u}$ and $\underline{u}$ be ordered coupling upper and lower solutions of (1). Then (1) has at least one solution $u$ in $[\underline{u}, \bar{u}]$.

Remark 3. Obviously, in the special case that $m=1$, Theorem 2 generalize the corresponding Amann [6, Theorem 2.1]. In addition, we would like to point out that Theorem 2 also generalizes Wang [9, Theorem 3.7.1], where the author developed the method of lower and upper solutions for the system (1) with linear boundary conditions.

The plan of the paper is as follows. In Section 2 we will develop the method of lower and upper solutions for elliptic system (1) and prove Theorem 2. Finally in Section 3, we will apply Theorem 2 to show the existence of positive solution of a juvenile-adult model.

\section{Lower and Upper Solutions Method}

Lemma 4. Let (H1) and (H2) hold. Then the system (1) is equivalent to the fixed point equation $u=T(u)$ in $[C(\bar{\Omega})]^{m}$. The map $T:[\underline{u}, \bar{u}] \subset[C(\bar{\Omega})]^{m} \rightarrow[C(\bar{\Omega})]^{m}$ is completely continuous; that is, $T$ is continuous and maps bounded set into compact set.

Proof. For every $(v, w)=\left(\left(v_{1}, \ldots, v_{m}\right),\left(w_{1}, \ldots, w_{m}\right)\right) \in$ $\left[C^{\alpha}(\bar{\Omega})\right]^{m} \times\left[C^{1+\alpha}(\Gamma)\right]^{m}$, it is well known $(\mathrm{cf} .[10,11])$ that the system

$$
\begin{aligned}
& L_{k} u_{k}=v_{k}, \quad x \in \Omega, \\
& \frac{\partial u_{k}}{\partial \mathbf{n}}+b_{k}(x) u_{k}=w_{k}, \quad x \in \Gamma, \\
& k=1,2, \ldots, m,
\end{aligned}
$$

has a unique solution $u=\left(u_{1}, \ldots, u_{m}\right)=: S(v, w) \epsilon$ $\left[C^{2+\alpha}(\bar{\Omega})\right]^{m}$ as well as in $\left[W_{p}^{2}(\Omega)\right]^{m}, 1<p<\infty$. Hence the Schauder estimates and the $L^{p}$-estimates take the form

$$
\begin{array}{r}
\left\|u_{k}\right\|_{C^{2+\alpha}(\bar{\Omega})} \leq C\left(\left\|v_{k}\right\|_{C^{\alpha}(\bar{\Omega})}+\left\|w_{k}\right\|_{C^{1+\alpha}(\Gamma)}\right), \\
k=1,2, \ldots, m, \\
\left\|u_{k}\right\|_{W_{p}^{2}(\Omega)} \leq C\left(\left\|v_{k}\right\|_{L^{p}(\Omega)}+\left\|w_{k}\right\|_{W_{p}^{1-1 / p}(\Gamma)}\right), \\
k=1,2, \ldots, m,
\end{array}
$$


respectively. Here and in the following $C$ denotes a positive constant (not necessarily the same in different formulas) which is independent of the functions appearing in these estimates. Hence (8) implies that $S:\left[C^{\alpha}(\bar{\Omega})\right]^{m} \times\left[C^{1+\alpha}(\Gamma)\right]^{m} \rightarrow$ $\left[C^{2+\alpha}(\bar{\Omega})\right]^{m}$ is a bounded linear operator. Since $[C(\bar{\Omega})]^{m} \hookrightarrow$ $\left[L_{p}(\Omega)\right]^{m}$ and $[C(\Gamma)]^{m} \hookrightarrow\left[L_{p}(\Gamma)\right]^{m}$ for every $p \in(1, \infty)$, Amann [6, Proposition 3.3] implies the existence of constant $C$ such that

$$
\begin{array}{r}
\left\|u_{k}\right\|_{W_{p}^{1}(\Omega)} \leq C\left(\left\|v_{k}\right\|_{L^{p}(\Omega)}+\left\|w_{k}\right\|_{L^{p}(\Gamma)}\right) \\
\leq C\left(\left\|v_{k}\right\|_{C(\bar{\Omega})}+\left\|w_{k}\right\|_{C(\Gamma)}\right), \\
k=1,2, \ldots, m,
\end{array}
$$

for every $(v, w) \in\left[C^{\alpha}(\bar{\Omega})\right]^{m} \times\left[C^{1+\alpha}(\Gamma)\right]^{m}$. Since the latter space is dense in $[C(\bar{\Omega})]^{m} \times[C(\Gamma)]^{m}$, the estimate (10) implies that $S$ has a unique continuous extension for each $p \in(1, \infty)$, denoted again by $S$, such that $S$ is also a bounded linear operator from $[C(\bar{\Omega})]^{m} \times[C(\Gamma)]^{m}$ to $\left[W_{p}^{1}(\Omega)\right]^{m}$.

Now, let $F$ and $G$ be the Nemytskii operators generated by the vector fields $\left(f_{1}, \ldots, f_{m}\right)$ and $\left(g_{1}, \ldots, g_{m}\right)$, respectively. Here and in the following we denote by $p$ an arbitrary, but fixed real number satisfying $p>N$. This implies in particular that $\left[W_{p}^{1}(\Omega)\right]^{m}$ is compactly embedded into $[C(\bar{\Omega})]^{m}$. We define $T:[\underline{u}, \bar{u}] \rightarrow\left[W_{p}^{1}(\Omega)\right]^{m}\left(\hookrightarrow \hookrightarrow[C(\bar{\Omega})]^{m}\right)$ by

$$
T(u)=S(F(u), G \circ \tau(u)),
$$

where $\tau$ denotes the usual trace operator. Then $T$ can be considered as a mapping of $[\underline{u}, \bar{u}]$ into $[C(\bar{\Omega})]^{m}$. It is obvious that every solution of the system (1) is a fixed point of $T$. Conversely, if $u \in[C(\bar{\Omega})]^{m}$ is a fixed point of $T$, then we can show that $u$ is also a solution of the system (1) by using the same methods as in the proof of Amann [6, Lemma 4.1].

Let $I=\prod_{k=1}^{m}\left[\min \underline{u}_{k}, \max \bar{u}_{k}\right]$. Then it is easy to see that $F:[\underline{u}, \bar{u}] \rightarrow[C(\bar{\Omega})]^{m}$ and $G: I_{\Gamma}:=\left\{u \in[C(\Gamma)]^{m}: u(\Gamma) \subset\right.$ $I\} \rightarrow[C(\Gamma)]^{m}$ are bounded and continuous. Moreover, $\tau$ : $[C(\bar{\Omega})]^{m} \rightarrow[C(\Gamma)]^{m}$ is a continuous linear operator such that $\tau([\underline{u}, \bar{u}]) \subset I_{\Gamma}$. Since $S$ is also a bounded linear operator from $[C(\bar{\Omega})]^{m} \times[C(\Gamma)]^{m}$ to $\left[W_{p}^{1}(\Omega)\right]^{m}$, and since $\left[W_{p}^{1}(\Omega)\right]^{m}$ is compactly embedded into $[C(\bar{\Omega})]^{m}$ for $p>N$, it follows that $T$ is completely continuous from $[\underline{u}, \bar{u}]$ to $[C(\bar{\Omega})]^{m}$.

Proof of Theorem 2. The regularity assumption ( $\mathrm{H} 1)$ for $f_{k}$ and $g_{k}$ implies the existence of positive constants $\sigma_{k}(k=$ $1,2, \ldots, m)$ such that

$$
\begin{aligned}
f_{k}\left(x, \bar{u}_{k},[v]_{k}\right)-f_{k}\left(x, v_{k},[v]_{k}\right) & \geq-\sigma_{k}\left(\bar{u}_{k}-v_{k}\right), \\
\forall \underline{u} & \leq v \leq \bar{u}, \forall x \in \bar{\Omega}, \\
f_{k}\left(x, v_{k},[v]_{k}\right)-f_{k}\left(x, \underline{u}_{k},[v]_{k}\right) & \geq-\sigma_{k}\left(v_{k}-\underline{u}_{k}\right), \\
\forall \underline{u} & \leq v \leq \bar{u}, \forall x \in \bar{\Omega}, \\
g_{k}\left(y, \bar{u}_{k},[v]_{k}\right)-g_{k}\left(y, v_{k},[v]_{k}\right) & \geq-\sigma_{k}\left(\bar{u}_{k}-v_{k}\right), \\
\forall \underline{u} & \leq v \leq \bar{u}, \forall y \in \Gamma, \\
g_{k}\left(y, v_{k},[v]_{k}\right)-g_{k}\left(y, \underline{u}_{k},[v]_{k}\right) & \geq-\sigma_{k}\left(v_{k}-\underline{u}_{k}\right), \\
\forall \underline{u} & \leq v \leq \bar{u}, \forall y \in \Gamma .
\end{aligned}
$$

Let

$$
M_{k}:=\sigma_{k}+\max \left\{\left\|a_{k}\right\|_{C(\bar{\Omega})},\left\|b_{k}\right\|_{C(\Gamma)}\right\} .
$$

Then the system (1) is equivalent to the system

$$
\begin{array}{r}
\left(L_{k}+M_{k}\right) u_{k}=f_{k}\left(x, u_{k},[u]_{k}\right)+M_{k} u_{k}, \quad x \in \Omega, \\
\frac{\partial u_{k}}{\partial \mathbf{n}}+b_{k}(x) u_{k}+M_{k} u_{k}=g_{k}\left(x, u_{k},[u]_{k}\right)+M_{k} u_{k}, \\
x \in \Gamma, k=1,2, \ldots, m .
\end{array}
$$

Moreover, $\underline{u}$ and $\bar{u}$ are also subsolution and supersolution for the system (17). Consequently, by Lemma 4 , system (17) is equivalent to the fixed point equation $u=T(u)$ in $[C(\bar{\Omega})]^{m}$, and $T:[\underline{u}, \bar{u}] \rightarrow[C(\bar{\Omega})]^{m}$ is completely continuous.

Now, let us show that $T:[\underline{u}, \bar{u}] \rightarrow[\underline{u}, \bar{u}]$.

It is clear that $[\underline{u}, \bar{u}]$ is a bounded closed convex subset of $[C(\bar{\Omega})]^{m}$. Let $v \in[\underline{u}, \bar{u}]$ and $u=T(v)$. Then $u$ and $v$ satisfy that

$$
\begin{array}{r}
L_{k} u_{k}+M_{k} u_{k}=f_{k}\left(x, v_{k},[v]_{k}\right)+M_{k} v_{k}, \quad x \in \Omega, \\
\frac{\partial u_{k}}{\partial \mathbf{n}}+b_{k}(x) u_{k}+M_{k} u_{k}=g_{k}\left(x, v_{k},[v]_{k}\right)+M_{k} v_{k}, \\
x \in \Gamma, k=1,2, \ldots, m .
\end{array}
$$

On the other hand, since $v \in[\underline{u}, \bar{u}]$, we have $\underline{u} \leq v \leq \bar{u}$ and so $[\underline{u}]_{k} \leq[v]_{k} \leq[\bar{u}]_{k}$, which together with Definition 1 yields that $\bar{u}$ verifies

$$
\begin{array}{r}
L_{k} \bar{u}_{k}+M_{k} \bar{u}_{k} \geq f_{k}\left(x, \bar{u}_{k},[v]_{k}\right)+M_{k} \bar{u}_{k}, \quad x \in \Omega, \\
\frac{\partial \bar{u}_{k}}{\partial \mathbf{n}}+b_{k}(x) \bar{u}_{k}+M_{k} \bar{u}_{k} \geq g_{k}\left(x, \bar{u}_{k},[v]_{k}\right)+M_{k} \bar{u}_{k}, \\
x \in \Gamma, k=1,2, \ldots, m .
\end{array}
$$

Let $w_{k}=\bar{u}_{k}-u_{k}$. Then we can easily conclude from (12), (14), (18), and (19) that

$$
\begin{aligned}
L_{k} w_{k}+M_{k} w_{k} \geq & f_{k}\left(x, \bar{u}_{k},[v]_{k}\right)-f_{k}\left(x, v_{k},[v]_{k}\right) \\
& +M_{k}\left(\bar{u}_{k}-v_{k}\right) \geq 0, \\
x \in & \Omega, k=1,2, \ldots, m, \\
\frac{\partial w_{k}}{\partial \mathbf{n}}+b_{k}(x) w_{k}+M_{k} w_{k} \geq & g_{k}\left(x, \bar{u}_{k},[v]_{k}\right)-g_{k}\left(x, v_{k},[v]_{k}\right) \\
& +M_{k}\left(\bar{u}_{k}-v_{k}\right) \geq 0, \\
x & \in \Gamma, k=1,2, \ldots, m .
\end{aligned}
$$

From the maximum principle for elliptic boundary value problems it follows that $w_{k} \geq 0$; that is, $u_{k} \leq \bar{u}_{k}$. Similarly, by using (13) and (15), we can obtain $\underline{u}_{k} \leq u_{k}$. Consequently, $T$ maps $[\underline{u}, \bar{u}]$ into itself, and the existence of a fixed point follows from Schauder fixed point theorem. 


\section{Application to a Juvenile-Adult Model}

In this section, we will apply the method of lower and upper solutions in Section 2 to study the existence of coexistence states of the following elliptic system describing two subpopulations of the same species competing for resources:

$$
\begin{array}{rr}
-\Delta u=a(x) v-c(x) u-e(x) & u(u+v), \\
x \in \Omega, \\
-\Delta v=b(x) u-d(x) v-f(x) v(u+v), \\
x \in \Omega, \\
\frac{\partial u}{\partial \mathbf{n}}+\alpha(x) u=g(x, u, v), & x \in \Gamma, \\
\frac{\partial v}{\partial \mathbf{n}}+\beta(x) v=h(x, u, v), & x \in \Gamma .
\end{array}
$$

System (21) arises from population dynamics where it models the steady-state solutions of the corresponding nonlinear evolution problem [12], where $u$ and $v$ represent the concentrations of the adult and juvenile populations, respectively. The function $a$ gives the rate at which juveniles become adults and $c$ corresponds to the death rate of adult population. As adults give birth to juveniles, the function $b$ corresponds to the birth rate of the population. Juveniles are lost both through death and through becoming adults, the function $d$ corresponds to this overall loss. The Laplacian operator shows the diffusive character of $u$ and $v$ within $\Omega$. By using fixed point theory and lower and upper solutions method, several authors have studied the existence of coexistence states of the system (21), subject to Dirichlet or Neumann boundary conditions; see, for example, [1316] and the references therein. We consider here the more general model (21), in which the boundary conditions may be interpreted as the conditions that the populations may pass through the boundary of the habitat. This is a mathematical model more closer to the reality.

In the rest of this section, we suppose that $\alpha$ and $\beta$ satisfy (H2), the coefficients $a, b, c, d, e$, and $f$ are positive functions in $C^{\alpha}(\bar{\Omega})$, and $g$ and $h$ are nonnegative functions defined on $\Gamma \times \mathbb{R}_{+}^{2}\left(\mathbb{R}_{+}=[0, \infty)\right)$ and satisfy $(\mathrm{H} 1)$. We will use the notation $u \gg 0$ if $u(x)>0$ for $x \in \bar{\Omega}$. Apparently, since $a(x)>0, b(x)>0$ for $x \in \Omega$, the system

$$
\begin{gathered}
-\Delta u=a(x) v-c(x) u, \quad x \in \Omega, \\
-\Delta v=b(x) u-d(x) v, \quad x \in \Omega, \\
\frac{\partial u}{\partial \mathbf{n}}+\alpha(x) u=0, \quad x \in \Gamma, \\
\frac{\partial v}{\partial \mathbf{n}}+\beta(x) v=0, \quad x \in \Gamma,
\end{gathered}
$$

is a linear cooperative system, for which we can give the following strong maximum principle.
Lemma 5. Let $u_{0}, v_{0} \in C^{2}(\Omega) \cap C^{1}(\bar{\Omega})$ be such that $u_{0} \gg$ $0, v_{0} \gg 0$ and

$$
\begin{gathered}
-\Delta u_{0} \geq a(x) v_{0}-c(x) u_{0}, \quad x \in \Omega, \\
-\Delta v_{0} \geq b(x) u_{0}-d(x) v_{0}, \quad x \in \Omega, \\
\frac{\partial u_{0}}{\partial \mathbf{n}}+\alpha(x) u_{0} \geq 0, \quad x \in \Gamma, \\
\frac{\partial v_{0}}{\partial \mathbf{n}}+\beta(x) v_{0} \geq 0, \quad x \in \Gamma,
\end{gathered}
$$

where equality does not hold in some of the equations in (23). Then (22) satisfies the strong maximum principle; that is, if $u, v \in C^{2}(\Omega) \cap C^{1}(\bar{\Omega})$ such that

$$
\begin{gathered}
-\Delta u \geq a(x) v-c(x) u, \quad x \in \Omega, \\
-\Delta v \geq b(x) u-d(x) v, \quad x \in \Omega, \\
\frac{\partial u}{\partial \mathbf{n}}+\alpha(x) u \geq 0, \quad x \in \Gamma, \\
\frac{\partial v}{\partial \mathbf{n}}+\beta(x) v \geq 0, \quad x \in \Gamma,
\end{gathered}
$$

then either (i) $u, v \equiv 0$ on $\Omega$ or (ii) $u \gg 0, v \gg 0$.

Proof. Otherwise, there exist $u_{1}, v_{1}$ not both identically zero satisfying (24) but not satisfying (ii) in the conclusion of the theorem. For $t \in[0,1]$, define $u_{t}=(1-t) u_{0}+t u_{1}$ and $v_{t}=$ $(1-t) v_{0}+t v_{1}$. Then there exists $t_{0}$ with $0<t_{0} \leq 1$ such that $u_{t}, v_{t} \gg 0$ for $0 \leq t<t_{0}$ and either $u_{t_{0}}$ or $v_{t_{0}}$ has a zero in $\bar{\Omega}$. Without loss of generality, we assume that there exists $x_{1} \in \bar{\Omega}$ such that $u_{t_{0}}\left(x_{1}\right)=0$. Then by (23) and (24) we have

$$
-\Delta u_{t_{0}} \geq a(x) v_{t_{0}}-c(x) u_{t_{0}}, \quad x \in \Omega
$$

That is,

$$
-\Delta u_{t_{0}}+c(x) u_{t_{0}} \geq a(x) v_{t_{0}} \geq 0, \quad x \in \Omega .
$$

On the other hand, we can also deduce from (23) and (24) that

$$
\frac{\partial u_{t_{0}}}{\partial \mathbf{n}}(x)+\alpha(x) u_{t_{0}}(x) \geq 0, \quad x \in \Gamma,
$$

and therefore the maximum principle for elliptic boundary value problems yields that $u_{t_{0}} \equiv 0$ or $u_{t_{0}} \gg 0$ on $\bar{\Omega}$. Since $u_{t_{0}}\left(x_{1}\right)=0$, we have that $u_{t_{0}} \equiv 0$, and then from (26) we get $v_{t_{0}} \equiv 0$. Since $u_{1}$ and $v_{1}$ are not both identically zero, $t_{0}<1$. Thus, as $u_{t_{0}}=\left(1-t_{0}\right) u_{0}+t_{0} u_{1} \equiv 0$ and $v_{t_{0}}=\left(1-t_{0}\right) v_{0}+t_{0} v_{1} \equiv$ 0 , we have, for $\theta:=\left(t_{0}-1\right) / t_{0}<0$,

$$
u_{1}=\theta u_{0}, \quad v_{1}=\theta v_{0} .
$$

This is impossible since $u_{0}, v_{0}$ satisfy (23) and $u_{1}, v_{1}$ satisfy (24).

Corollary 6. Assume that $a(x)-c(x)<0$ and $b(x)-d(x)<0$ for all $x \in \Omega$. Then the system (22) verifies the strong maximum principle. 
Proof. We may get the desired results by using Theorem 9 and choosing $u_{0} \equiv v_{0} \equiv M$, where $M$ is any positive constant.

Lemma 7. The cooperative system (22) has a principal eigenvalue; that is, there exists $\Lambda \in \mathbb{R}$ and $u_{0}, v_{0} \in C^{2}(\Omega) \cap C^{1}(\bar{\Omega})$ such that $u_{0} \gg 0, v_{0} \gg 0$ and

$$
\begin{gathered}
-\Delta u_{0}+c(x) u_{0}-a(x) v_{0}=\Lambda u_{0}, \quad x \in \Omega, \\
-\Delta v_{0}-b(x) u_{0}+d(x) v_{0}=\Lambda v_{0}, \quad x \in \Omega, \\
\frac{\partial u_{0}}{\partial \mathbf{n}}+\alpha(x) u_{0}=0, \quad x \in \Gamma, \\
\frac{\partial v_{0}}{\partial \mathbf{n}}+\beta(x) v_{0}=0, \quad x \in \Gamma .
\end{gathered}
$$

Proof. Obviously, system (22) can be equivalently rewritten as

$$
\begin{aligned}
& \mathfrak{Q} U-A(x) U=0, \quad x \in \Omega, \\
& \frac{\partial u}{\partial \mathbf{n}}+\alpha(x) u=0, \quad x \in \Gamma, \\
& \frac{\partial v}{\partial \mathbf{n}}+\beta(x) v=0, \quad x \in \Gamma,
\end{aligned}
$$

where

$$
\begin{aligned}
& U=\left(\begin{array}{l}
u \\
v
\end{array}\right), \quad \mathfrak{L}=\left(\begin{array}{cc}
-\Delta & 0 \\
0 & -\Delta
\end{array}\right), \\
& A(x)=\left(\begin{array}{cc}
-c(x) & a(x) \\
b(x) & -d(x)
\end{array}\right) .
\end{aligned}
$$

Let $X=\left\{U \in C^{2+\alpha}(\bar{\Omega}) \times C^{2+\alpha}(\bar{\Omega}): \partial u / \partial \mathbf{n}+\alpha(x) u=\right.$ $0, \partial v / \partial \mathbf{n}+\beta(x) v=0\}$ and $Y=C^{\alpha}(\bar{\Omega}) \times C^{\alpha}(\bar{\Omega})$. Then $\mathfrak{L}$ maps $X$ into $Y$. It is well known that if $M>0$ is sufficiently large, then $\mathbf{Q}-A(x)+M: X \rightarrow Y$ is invertible such that $(\mathbf{Q}-$ $A(x)+M)^{-1}$ is compact. Moreover, if $M$ is chosen sufficiently large such that $a(x)-c(x)<M$ and $b(x)-d(x)<M$ for all $x \in \Omega$, then Corollary 6 yields that $(\mathcal{L}-A(x)+M)^{-1}$ is strongly positive. It follows from the Krein-Rutman theorem that $(\mathbb{L}-A(x)+M)^{-1}$ has a positive principal eigenvalue, which is denoted by $\mu$. Therefore, there exists $\widetilde{U}=\left(\frac{\widetilde{u}}{\widetilde{v}}\right) \in Y \times Y$ with $\widetilde{u} \gg 0, \widetilde{v} \gg 0$ such that $(\mathbb{L}-A(x)+M)^{-1} \widetilde{U}=\mu \widetilde{U}$. Consequently, $(\mathbb{Q}-A(x)) \widetilde{U}=(1 / \mu-M) \widetilde{U}$ and so $\mathfrak{Q}-A(x)$ has a principal eigenvalue $\lambda_{1}(\mathfrak{L}-A(x))$.

Proposition 8. Assume that $A_{1}(x)=\left(a_{i j}^{1}(x)\right)_{2 \times 2}$ and $A_{2}(x)=$ $\left(a_{i j}^{2}(x)\right)_{2 \times 2}$ are cooperative matrices. If $A_{1}(x) \geq A_{2}(x)$, that is, $a_{i j}^{1}(x) \geq a_{i j}^{2}(x)$ for all $x \in \Omega$ and $i, j=1,2$ but $A_{1}(x) \neq \equiv A_{2}(x)$, then $\lambda_{1}\left(\mathfrak{L}-A_{1}(x)\right)<\lambda_{1}\left(\mathfrak{L}-A_{2}(x)\right)$.
Proof. By Lemma 7, there exists $U_{1}=\left(\begin{array}{c}u_{1} \\ v_{1}\end{array}\right) \in X$ so that $u_{1} \gg$ $0, v_{1} \gg 0$ and $\left[\mathfrak{L}-A_{1}(x)-\lambda_{1}\left(\mathfrak{L}-A_{1}(x)\right)\right] U_{1}=0$. Then

$$
\begin{aligned}
{[\mathfrak{Q}-} & \left.A_{2}(x)-\lambda_{1}\left(\mathfrak{L}-A_{1}(x)\right)\right] U_{1} \\
= & {\left[\mathfrak{L}-A_{1}(x)-\lambda_{1}\left(\mathfrak{L}-A_{1}(x)\right)\right] U_{1} } \\
& +\left(A_{1}(x)-A_{2}(x)\right) U_{1}=\left(A_{1}(x)-A_{2}(x)\right) U_{1} \geq 0,
\end{aligned}
$$

but $\left(A_{1}(x)-A_{2}(x)\right) U_{1} \not \equiv 0$, and from Lemma 5 we know that system $\left[\mathbb{L}-A_{2}(x)-\lambda_{1}\left(\mathfrak{L}-A_{1}(x)\right)\right] U=0$ satisfies the strong maximum principle. Hence if $\gamma$ denotes the principal eigenvalue of the system $\mathfrak{Q}-A_{2}(x)-\lambda_{1}\left(\mathfrak{L}-A_{1}(x)\right) I$, then it follows easily that $\gamma>0$. Clearly, $\mathfrak{Q}-A_{2}(x)$ has principal eigenvalue $\lambda_{1}\left(\mathfrak{L}-A_{2}(x)\right)=\lambda_{1}\left(\mathfrak{L}-A_{1}(x)\right)+\gamma>\lambda_{1}(\mathfrak{L}-$ $A_{1}(x)$.

For each positive function $\xi \in C^{\alpha}(\bar{\Omega})$, we denote by $\bar{\xi}$ and $\xi$ the maximum and minimum of $\xi$ in $\bar{\Omega}$, respectively. Let

$$
\begin{gathered}
\alpha_{0}=\min _{x \in \Gamma} \alpha(x), \quad \beta_{0}=\min _{x \in \Gamma} \beta(x), \\
M=\max \left\{\frac{\bar{a}}{\underline{e}}, \frac{\bar{b}}{\underline{f}}\right\} .
\end{gathered}
$$

Then they are all positive constants. Applying Theorem 2, we will prove the following existence results of positive solutions of system (21).

Theorem 9. Suppose that $g, h: \Gamma \times \mathbb{R}_{+}^{2} \rightarrow \mathbb{R}_{+}$satisfy

$$
\begin{array}{ll}
g(x, M, y) \leq M \alpha_{0} & \forall(x, y) \in \Gamma \times[0, M] ; \\
h(x, z, M) \leq M \beta_{0} & \forall(x, z) \in \Gamma \times[0, M] .
\end{array}
$$

If $\lambda_{1}(\mathbb{L}-A(x))<0$, then system (21) has at least one positive solution.

Proof. Assume $\lambda_{1}(\mathbb{Q}-A(x))<0$. Then there exists $\Phi=\left(\begin{array}{l}\phi_{1} \\ \phi_{2}\end{array}\right)$ with $\phi_{1} \gg 0, \phi_{2} \gg 0$ such that $(\mathfrak{L}-A(x)) \Phi=\lambda_{1}(\mathfrak{L}-A(x)) \Phi$; that is,

$$
\begin{aligned}
-\Delta \phi_{1}= & a(x) \phi_{2}-c(x) \phi_{1}+\lambda_{1}(\mathfrak{\Omega}-A(x)) \phi_{1}, \\
x \in \Omega, & \\
-\Delta \phi_{2}= & b(x) \phi_{1}-d(x) \phi_{2}+\lambda_{1}(\mathfrak{Q}-A(x)) \phi_{2}, \\
x \in \Omega, & \\
& \frac{\partial \phi_{1}}{\partial \mathbf{n}}+\alpha(x) \phi_{1}=0, \quad x \in \Gamma, \\
& \frac{\partial \phi_{2}}{\partial \mathbf{n}}+\beta(x) \phi_{2}=0, \quad x \in \Gamma .
\end{aligned}
$$

Let $U^{*}=\left(\begin{array}{c}u^{*} \\ v^{*}\end{array}\right)=\left(\begin{array}{c}M \\ M\end{array}\right)$ and $U_{*}=\left(\begin{array}{c}u_{*} \\ v_{*}\end{array}\right)=\varepsilon\left(\begin{array}{c}\phi_{1} \\ \phi_{2}\end{array}\right)$, where $M$ is given as in (33). In the following we will show that $U^{*}$ and $U_{*}$ satisfy the hypotheses of Theorem 2 if $M$ is defined as above and $\varepsilon>0$ is chosen sufficiently small. 
By the definition of $M$, we have $a(x)-e(x) M \leq 0$ for all $x \in \Omega$, and so

$$
\begin{array}{r}
c(x) M \geq 0 \geq(a(x)-e(x) M) v-e(x) M^{2}, \\
\forall 0 \leq v \leq M .
\end{array}
$$

In other words, we get

$$
\begin{array}{r}
-\Delta u^{*}+c(x) u^{*} \geq a(x) v-e(x) u^{*}\left(u^{*}+v\right), \\
\forall 0 \leq v \leq M .
\end{array}
$$

Moreover, from (33) it follows that

$$
\begin{array}{r}
\frac{\partial u^{*}}{\partial \mathbf{n}}+\alpha(x) u^{*}=\alpha(x) M \geq \alpha_{0} M \\
\geq g(x, M, v)=g\left(x, u^{*}, v\right), \\
\forall 0 \leq v \leq M .
\end{array}
$$

Similarly, we can deduce from (35) that

$$
\begin{array}{r}
-\Delta v^{*}+d(x) v^{*} \geq b(x) u-f(x) v^{*}\left(v^{*}+u\right), \\
\forall 0 \leq u \leq M, \\
\frac{\partial v^{*}}{\partial \mathbf{n}}+\beta(x) v^{*}=\beta(x) M \geq \beta_{0} M \\
\geq h(x, u, M)=h\left(x, u, v^{*}\right), \\
\forall 0 \leq u \leq M .
\end{array}
$$

Let $\widehat{\varepsilon}=\min \left\{\underline{a} / \bar{e} \bar{\phi}_{1}, \underline{b} / \bar{f} \bar{\phi}_{2}\right\}$. Then, for any $\varepsilon \in(0, \widehat{\varepsilon})$, we have $a(x)-\varepsilon e(x) \phi_{1}(x) \geq 0$ and $b(x)-\varepsilon f(x) \phi_{2}(x) \geq 0$ for all $x \in \Omega$. Hence when $\varepsilon<\widehat{\varepsilon}$ and $\varepsilon \phi_{2} \leq v \leq M$, we get

$$
\begin{aligned}
&-\Delta u_{*}-a(x) v+c(x) u_{*}+e(x) u_{*}\left(u_{*}+v\right) \\
&=-\varepsilon \Delta \phi_{1}-a(x) v+c(x) \varepsilon \phi_{1}+e(x) \varepsilon \phi_{1}\left(\varepsilon \phi_{1}+v\right) \\
&=\varepsilon\left(-\Delta \phi_{1}-a(x) \phi_{2}+c(x) \phi_{1}\right) \\
& \quad+a(x)\left(\varepsilon \phi_{2}-v\right)+e(x) \varepsilon \phi_{1}\left(\varepsilon \phi_{1}+v\right) \\
&=\varepsilon \lambda_{1}(\mathbb{Q}-A(x)) \phi_{1}+\varepsilon a(x) \phi_{2} \\
&-\left(a(x)-e(x) \varepsilon \phi_{1}\right) v+\varepsilon^{2} e(x) \phi_{1}^{2} \\
& \leq \varepsilon \lambda_{1}(\mathbb{L}-A(x)) \phi_{1}+\varepsilon a(x) \phi_{2} \\
&-\left(a(x)-e(x) \varepsilon \phi_{1}\right) \varepsilon \phi_{2}+\varepsilon^{2} e(x) \phi_{1}^{2} \\
&= \varepsilon \lambda_{1}(\mathbb{R}-A(x)) \phi_{1}+\varepsilon^{2} e(x) \phi_{1}\left(\phi_{1}+\phi_{2}\right)<0,
\end{aligned}
$$

when $\varepsilon$ is sufficiently small; that is,

$$
\begin{array}{r}
-\Delta u_{*}+c(x) u_{*}<a(x) v-e(x) u_{*}\left(u_{*}+v\right), \\
\forall \varepsilon \phi_{2} \leq v \leq M
\end{array}
$$

Furthermore, since the function $g$ is nonnegative, we have that

$$
\frac{\partial u_{*}}{\partial \mathbf{n}}+\alpha(x) u_{*}=\varepsilon\left(\frac{\partial \phi_{1}}{\partial \mathbf{n}}+\alpha(x) \phi_{1}\right)=0 \leq g\left(x, u_{*}, v\right),
$$

$$
\forall \varepsilon \phi_{2} \leq v \leq M
$$

Applying the similar discussions as above, we can also prove that

$$
\begin{aligned}
-\Delta v_{*}+d(x) v_{*}<b(x) u-f(x) v_{*}\left(v_{*}+u\right), \\
\forall \varepsilon \phi_{1} \leq u \leq M, \\
\frac{\partial v_{*}}{\partial \mathbf{n}}+\beta(x) v_{*}=\varepsilon\left(\frac{\partial \phi_{2}}{\partial \mathbf{n}}+\beta(x) \phi_{2}\right) \\
=0 \leq h\left(x, u, v_{*}\right), \quad \forall \varepsilon \phi_{1} \leq u \leq M .
\end{aligned}
$$

Consequently, it follows from Theorem 2 that system (21) has at least one positive solution $U$ in $\left[U_{*}, U^{*}\right]$.

Remark 10. It is worth remarking that the results of Theorem 9 remain true if we use the weaker assumption $g, h$ : $\Gamma \times \mathbb{R}_{+}^{2} \rightarrow \mathbb{R}_{+}$and

$$
\begin{gathered}
g(x, y, z) \geq 0, \quad h(x, y, z) \geq 0, \\
\forall x \in \Gamma,(y, z) \in[0, M] \times[0, M] .
\end{gathered}
$$

Remark 11. In the special case $g \equiv h \equiv 0$ on $\Gamma \times \mathbb{R}_{+}^{2}$, the assumptions (34) and (35) are fulfilled. From Proposition 8 and similar argument used in the proof of [14, Theorem 3.2], we may show that the condition $\lambda_{1}(\mathbb{R}-A(x))<0$ is also necessary for the existence of positive solutions of system (21). Consequently, if $g \equiv h \equiv 0$ on $\Gamma \times \mathbb{R}_{+}^{2}$, then system (21) has a positive solution if and only if $\lambda_{1}(\mathfrak{L}-A(x))<0$. Hence Theorem 9 generalizes [14, Theorem 3.2] and [15, Corollary 18] in which only the problem with linear boundary conditions was considered.

\section{Conflict of Interests}

The authors declare that there is no conflict of interests regarding the publication of this paper.

\section{Acknowledgments}

This work is supported by the NSFC (no. 11361054 and no. 11201378), SRFDP (no. 20126203110004), and Gansu provincial National Science Foundation of China (no. 1208RJZA258).

\section{References}

[1] H. Brézis, "Problèmes unilatéraux," Journal de Mathématiques Pures et Appliquées, vol. 51, pp. 1-168, 1972.

[2] H. Brill, "Eine stark nichtlineare elliptische Gleichung unter einer nichtlinearen Randbedingung," Zeitschrift für Angewandte Mathematik und Mechanik, Ingenieurwissenschaftliche Forschungsarbeiten, vol. 56, no. 3, pp. T243-T244, 1976. 
[3] J. P. Dias, "Un théorème de Sturm-Liouville pour une classe d'opérateurs non linéaires maximaux monotones," Journal of Mathematical Analysis and Applications, vol. 47, no. 2, pp. 400405, 1974

[4] J. M. Cushing, "Nonlinear Steklov problems on the unit circle," Journal of Mathematical Analysis and Applications, vol. 38, no. 3, pp. 766-783, 1972.

[5] K. Klingelhöfer, "Nonlinear harmonic boundary value problems. I," Archive for Rational Mechanics and Analysis, vol. 31, no. 5, pp. 364-371, 1968.

[6] H. Amann, "Nonlinear elliptic equations with nonlinear boundary conditions," in New Developments in Differential Equations, pp. 43-63, North-Holland, Amsterdam, 1976.

[7] H. Amann, "On the existence of positive solutions of nonlinear elliptic boundary value problems," Indiana University Mathematics Journal, vol. 21, no. 2, pp. 125-146, 1971.

[8] P. Hess, "On the solvability of nonlinear elliptic boundary value problems," Indiana University Mathematics Journal, vol. 25, no. 5, pp. 461-466, 1976.

[9] M. Wang, Nonlinear Elliptic Equations, Science Press, Beijing, Germany, 2010, Chinese.

[10] O. A. Ladyzhenskaya and N. N. Ural'cprimetseva, Linear and Quasilinear Elliptic Equations, Academic Press, New York, NY, USA, 1968.

[11] C. Miranda, Partial Differential Equations of Elliptic Type, Springer, Berlin, Germany, 1970.

[12] O. Arino and J.-A. Montero-Sánchez, "Optimal control of a nonlinear elliptic population system," Proceedings of the Edinburgh Mathematical Society II, vol. 43, no. 2, pp. 225-241, 2000.

[13] A. Cañada, P. Magal, and J. A. Montero, "Optimal control of harvesting in a nonlinear elliptic system arising from population dynamics," Journal of Mathematical Analysis and Applications, vol. 254, no. 2, pp. 571-586, 2001.

[14] K. J. Brown and Y. Zhang, "On a system of reaction-diffusion equations describing a population with two age groups," Journal of Mathematical Analysis and Applications, vol. 282, no. 2, pp. 444-452, 2003.

[15] S. M. Bouguima, S. Fekih, and W. Hennaoui, "Spacial structure in a juvenile-adult model," Nonlinear Analysis, Real World Applications, vol. 9, no. 3, pp. 1184-1201, 2008.

[16] O. Henaoui, "An elliptic system modeling two subpopulations," Nonlinear Analysis, Real World Applications, vol. 13, no. 6, pp. 2447-2458, 2012. 


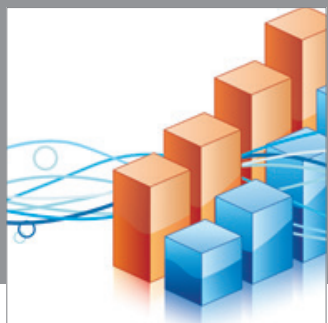

Advances in

Operations Research

mansans

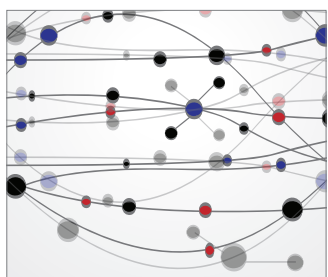

The Scientific World Journal
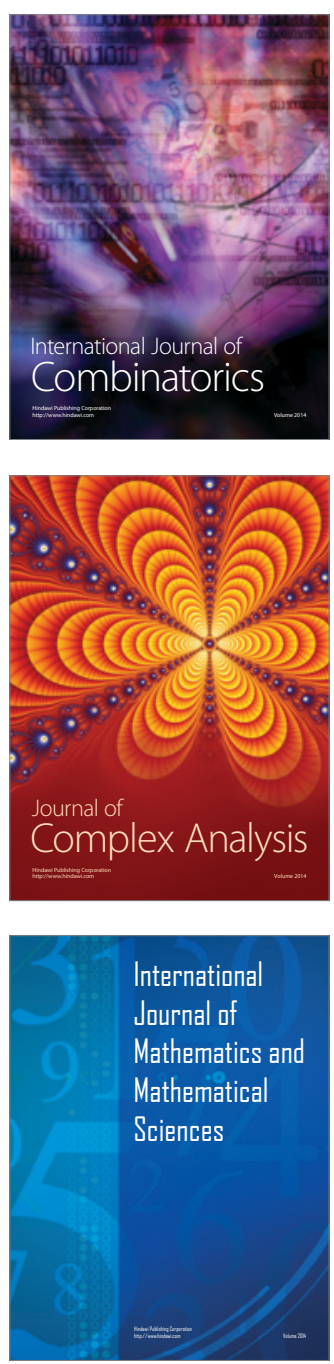
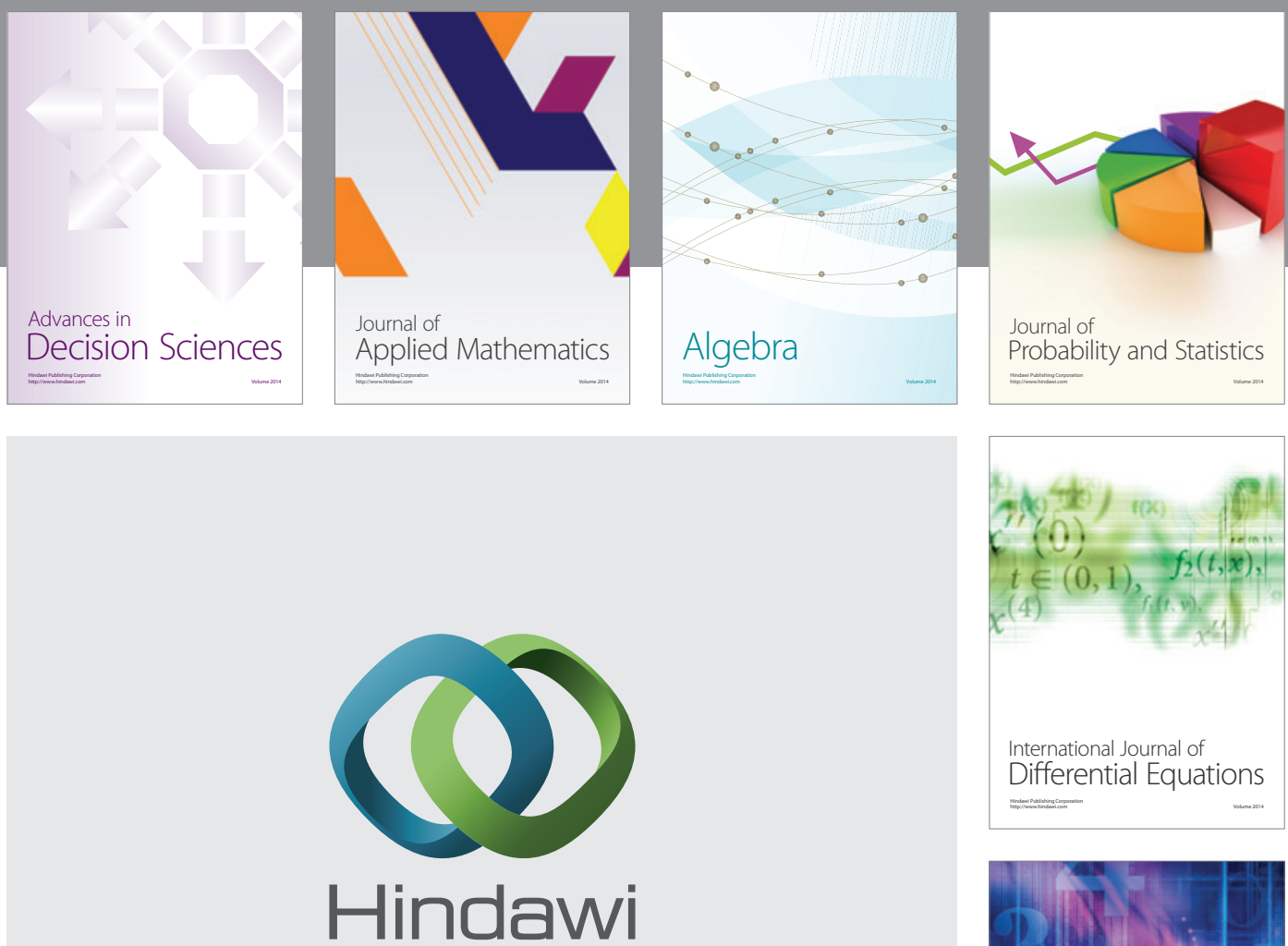

Submit your manuscripts at http://www.hindawi.com
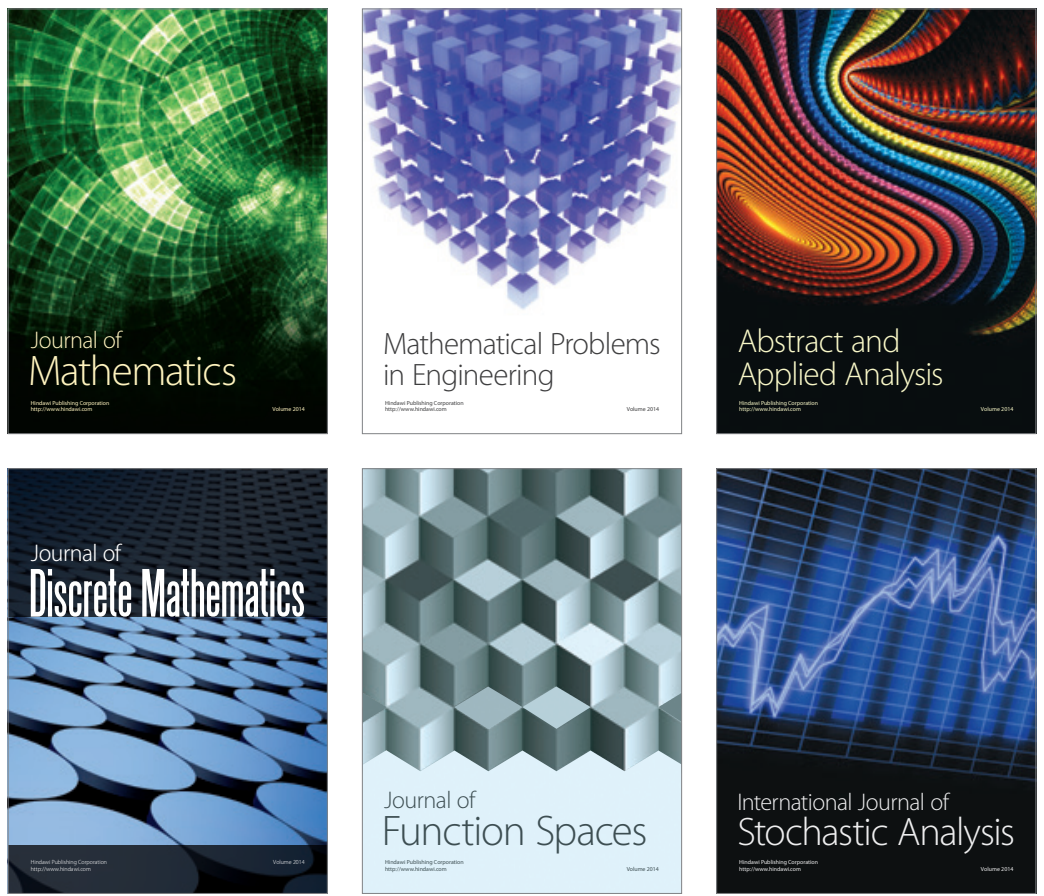

Journal of

Function Spaces

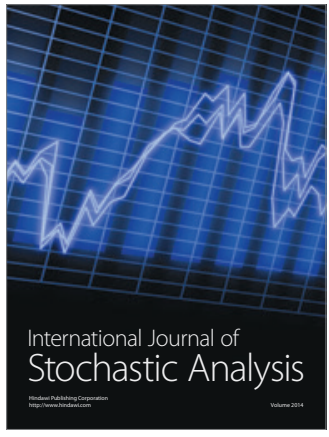

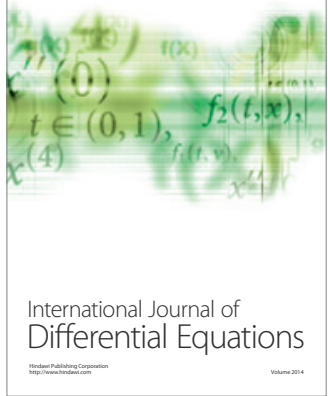
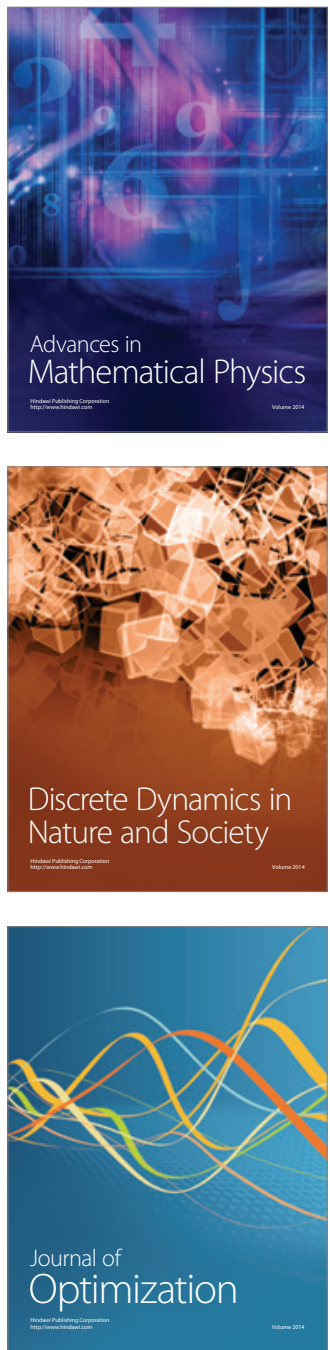